\title{
A THEOREM ON SUBHARMONIC FUNCTIONS
}

WALTER RUDIN

A real function $u$ is said to be subharmonic in a finite plane domain $D$ if $[2, \text { p. 333 }]^{1}$

(a) $u$ is upper semi-continuous in $D$;

(b) $u(P)<+\infty$ for $P$ in $D$;

(c) $u(P)$ is finite in a dense set in $D$;

(d) for every domain $G$ with boundary $B$, such that $G+B \subset D$, the inequality $u(P) \leqq h(P)$ on $B$ implies $u(P) \leqq h(P)$ in $G$ for every function $h$ which is harmonic in $G$ and continuous on $G+B$.

We use the notation [3]

$$
\Delta_{r} f(M)=\frac{1}{2 \pi} \int_{0}^{2 \pi} f(r, \theta) d \theta-f(M),
$$

where $(r, \theta)$ is a polar coordinate system with pole at $M$, provided that $f(r, \theta) \in L$ on $[0,2 \pi]$. If $\Delta_{r} f(M)$ is defined for all sufficiently small $r$ we put

$$
\Lambda^{*} f(M)=\limsup _{r \rightarrow 0} 4 \Delta_{r} f(M) / r^{2} .
$$

$\Lambda f(M)$ and $\Lambda_{*} f(M)$ are defined likewise, with lim and lim inf in place of lim sup.

(3) Theorem. Suppose

(i) the function $u$ is upper semi-continuous in the finite plane domain $D$;

(ii) $\Lambda^{*} u(P) \geqq 0$ for $P$ in $D-E$, where $E$ is at most countable;

(iii) $\lim \sup _{r \rightarrow 0} \Delta_{r} u(P) / r \geqq 0$ for $P$ in $E$.

Then $u$ is subharmonic in $D$.

The special case of the theorem in which $E$ is vacuous is known $[1$, p. 14]. At the end of the present paper, (3) is used to extend the results of $[3]$ and $[4]$.

Proof of (3). The existence of $\Lambda^{*} u(P)$ implies that $u \in L$ on every sufficiently small circle about $P$. Hence condition (c) is satisfied. By (ii) and (iii), condition (b) evidently also holds.

It suffices to prove (3) if (ii) is replaced by

$$
\Lambda^{*} u(P)>0
$$$$
(P \text { in } D-E) \text {. }
$$

Presented to the Society, December 29, 1949; received by the editors December 9 , 1949 and, in revised form, February 27, 1950.

${ }^{1}$ Numbers in brackets refer to the bibliography at the end of the paper. 
For, having established the theorem for this case, we put

$$
u_{m}(P)=u_{m}(x, y)=u(x, y)+x^{2} / m \quad(m=1,2,3, \ldots ; P \text { in } D),
$$

so that $\Lambda^{*} u_{m}(P)=\Lambda^{*} u(P)+2 / m>0(m=1,2,3, \cdots ; P$ in $D-E)$, and $u_{m}$ satisfies (iii). Letting $m \rightarrow \infty$, we see that $u$ is the limit of a monotonically decreasing sequence of subharmonic functions, and is therefore itself subharmonic [2, p. 335].

We assume (ii') to hold. For $P$ in $D$, we put

$$
\mu(u ; P)=\operatorname{liv}_{0 \leqq \theta<2 \pi} \limsup _{r \rightarrow 0} \frac{u(r, \theta)-u(P)}{r}
$$

where 1.u.b. stands for least upper bound, and $(r, \theta)$ is a polar coordinate system with pole at $P$. If the theorem is false, there exists a domain $G$ with boundary $B, G+B \subset D$, and a function $h$, continuous on $G+B$, harmonic in $G$, and such that

$$
\begin{array}{lr}
u(P) \leqq h(P) & (P \text { on } B), \\
u(Q)>h(Q) & \text { for some } Q \text { in } G .
\end{array}
$$

We may assume that $G$ is bounded. (In fact, we can assume that $G$ is circular [2, p. 334], but this would not simplify the proof.) Furthermore, we can choose $h$ such that

$$
|\nabla h(P)| \neq \mu(u ; P)
$$

where $\nabla h$ is the gradient of $h$. For suppose (7) is false. Since $G$ is bounded, we can choose a coordinate system $(x, y)$ such that $x>0$ for all points of $G$. Clearly there exists $\delta>0$ such that (5) and (6) hold if $h(P)$ is replaced by $h_{1}(P)=h(P)+\epsilon x(P$ in $G+B ; 0 \leqq \epsilon \leqq \delta)$. Since $E$ is at most countable, we can choose $\epsilon$ in $[0, \delta]$ such that (7) is also satisfied by $h_{1}$.

Put $w(P)=u(P)-h(P)$. By (5) and (6), the upper semi-continuous function $w$ attains its maximum at a point $M$ in $G$. It follows that

$$
\Lambda^{*} u(M)=\Lambda^{*}(w(M)+h(M))=\Lambda^{*} w(M) \leqq 0,
$$

which contradicts (ii') if $M \in D-E$.

If $M \in E, \lim \sup _{r \rightarrow 0} \Delta_{r} w(M) r \geqq 0$. Since $w(r, \theta) \leqq w(M)$ for sufficiently small $r$, an application of Fatou's theorem gives

$$
\begin{aligned}
0 & \leqq \int_{0}^{2 \pi} \liminf _{r \rightarrow 0} \frac{w(M)-w(r, \theta)}{r} d \theta \\
& \leqq \liminf _{r \rightarrow 0} \int_{0}^{2 \pi} \frac{w(M)-w(r, \theta)}{r} d \theta \leqq 0
\end{aligned}
$$


It follows that

$$
\begin{array}{ll}
\limsup _{r \rightarrow 0} \frac{w(r, \theta)-w(M)}{r}=0 & \text { for almost all } \theta, \\
\limsup _{r \rightarrow 0} \frac{w(r, \theta)-w(M)}{r} \leqq 0 & (0 \leqq \theta<2 \pi) .
\end{array}
$$

Therefore, putting $\Theta=i \cos \theta+j \sin \theta$, where $i, j$ are unit vectors in the $x, y$ directions, we have

$$
\begin{aligned}
& \limsup _{r \rightarrow 0} \frac{u(r, \theta)-u(M)}{r}=\Theta \cdot \nabla h(M) \quad \text { for almost all } \theta, \\
& \limsup _{r \rightarrow 0} \frac{u(r, \theta)-u(M)}{r} \leqq \Theta \cdot \nabla h(M) \quad(0 \leqq \theta<2 \pi) .
\end{aligned}
$$

Hence $\mu(u ; M)=|\nabla h(M)|$, which contradicts (7). This completes the proof.

The theorem just proved enables us to derive the conclusion of Theorem I of [3] from weaker hypotheses. We obtain the following theorem.

(8) Theorem. Let $F$ be continuous in the finite plane domain $D$. Let $Z$ be a closed and bounded set of capacity zero. Suppose

(i') $\Lambda^{*} F(P)>-\infty$ on $D-D \cdot Z-E_{1}$, where $E_{1}$ is at most countable, and $\lim \sup _{r \rightarrow 0} \Delta_{r} F(P) / r \geqq 0$ on $E_{1}$;

(i') $\Lambda_{*} F(P)<+\infty$ on $D-D \cdot Z-E_{2}$, where $E_{2}$ is at most countable, and $\lim \inf _{r \rightarrow 0} \Delta_{r} F(P) / r \leqq 0$ on $E_{2}$;

(ii) there exists a function $y$, defined in $D$, such that $y \in L$ on every closed subset of $D$, and such that $y(P) \leqq \Lambda^{*} F(P)$ for $P$ in $D$.

Then, for all bounded domains $R$ whose closure is contained in $D$, and at almost all points $P$ of $R$,

$$
F(P)=-\frac{1}{2 \pi} \iint_{R} \Lambda F(Q) g(P, Q) d Q+H(P),
$$

where $g(P, Q)$ is Green's function for $R$, and $H$ is harmonic in $R$ and assumes the values of $F$ on the boundary of $R$.

In [3], this theorem is proved for the case $E_{1}=E_{2}=0$. To prove (8), we use the notation

$$
\Omega f(P)=-\frac{1}{2 \pi} \iint_{R} f(Q) g(P, Q) d Q,
$$

as in [3], and put 


$$
W(P)=F(P)-\Omega u(P) \quad(P \in R),
$$

where $u$ is an upper semi-continuous function associated with $y$ in the sense of the Vitali-Carathéodory theorem. As in (4.2.2) of [3], we have now

$$
\Lambda^{*} W(P) \geqq 0 \quad\left(P \in R-R \cdot Z-R \cdot E_{1}\right) .
$$

Since $u$ is bounded above in $R$, we have, by (3.5.1) of [3],

$$
\begin{aligned}
\limsup _{r \rightarrow 0} \frac{1}{r} \Delta_{r} \Omega u(P) & =\limsup _{r \rightarrow 0} \frac{1}{2 \pi r} \iint_{P Q<r} u(Q) \log \frac{r}{P Q} d Q \\
& \leqq \limsup _{r \rightarrow 0} \frac{m r}{4}=0 \quad(P \in R),
\end{aligned}
$$

where $m$ is an upper bound of $u$ in $R$. Hence

$$
\begin{aligned}
\limsup _{r \rightarrow 0} \frac{1}{r} \Delta_{r} W(P) & \\
& \geqq \limsup _{r \rightarrow 0} \frac{1}{r} \Delta_{r} F(P)-\limsup _{r \rightarrow 0} \frac{1}{r} \Delta_{r} \Omega u(P) \geqq 0
\end{aligned}
$$

for $P$ on $R \cdot E_{1}$. By (9), (11), and (3), $W$ is subharmonic in the domain $R-R \cdot Z$. The proof then proceeds as in 4.2 of [3].

The hypotheses of Theorem II of [3] and of Theorems 2.6, 3.9, and 4.2 of [4] may be weakened in the same manner.

Finally, we wish to state the following analogue of (3).

(12) Theorem. Suppose

(i) the function $f$ is upper semi-continuous in the segment $I=(a, b)$;

(ii) $\lim \sup _{h \rightarrow 0}\{f(x+h)+f(x-h)-2 f(x)\} / h^{2} \geqq 0$ for $x$ in $I-E$, where $E$ is at most countable;

(iii) $\lim \sup _{h \rightarrow 0}\{f(x+h)+f(x-h)-2 f(x)\} / h \geqq 0$ for $x$ in $E$. Then $f$ is convex in $(a, b)$.

\section{BIBLIOGRAPHY}

1. T. Rad6, Subharmonic functions, Ergebnisse der Mathematik, vol. 5, no. 1, Berlin, 1925.

2. F. Riesz, Sur les fonctions subharmoniques et leur rapport à la theorie du potentiel, Acta Math. vol. 48 (1926) pp. 329-343.

3. W. Rudin, Integral representation of continuous functions, Trans. Amer. Math. Soc. vol. 68 (1950) pp. 278-286.

4. - Uniqueness theory for Laplace series, Trans. Amer. Math. Soc. vol. 68 (1950) pp. 287-303.

DUKE UNIVERSITY 